\title{
Felicidade e Indicadores de Psicopatologia em Brasileiros
}

\author{
Cristian Zanon \\ Universidade Federal do Rio Grande do Sul, Porto Alegre-RS, Brasil
}

\section{RESUMO}

Este estudo avalia as relações de satisfação de vida com depressão, ansiedade e estresse. Tais associações não foram ainda, conjuntamente, investigadas no contexto brasileiro. Foram participantes 371 universitários (64\% mulheres) que responderam à ESV e à Escala de Depressão, Ansiedade e Estresse. Correlações indicaram associação moderada de satisfação de vida com depressão $(r=-0,41)$ e pequenas com ansiedade $(r=-0,22)$ e estresse $(r=-0,25)$. Um modelo de regressão múltipla foi usado para avaliar o papel preditivo de depressão, ansiedade e estresse sobre a satisfação de vida. Esse modelo predisse $17 \%$ da variância total, sendo que apenas depressão $(\beta=-0,51)$ foi estatisticamente significativa. Esses resultados produzem evidências de validade para a ESV e são explorados em termos de possíveis implicações clínicas para o desenvolvimento de estratégias de aumento da felicidade.

Palavras-chave: satisfação de vida; bem-estar subjetivo; depressão; ansiedade; estresse.

\section{ABSTRACT - Happiness and Psychopathology Indicators in Brazilians}

This study evaluates the relationship between life satisfaction and depression, anxiety and stress. These associations have not been jointly investigated in the Brazilian context. A sample of 371 university students (64\% women) participated, each responding to the Life Satisfaction Scale (LSV) and the Depression, Anxiety and Stress Scale. Correlations indicated moderate association of life satisfaction with depression $(r=-0.41)$, and small associations with anxiety $(r=-0.22)$ and stress $(r=-0.25)$. A multiple regression model was used to assess the predictive role of depression, anxiety, and stress on life satisfaction. This model predicted $17 \%$ of the total variance, and only depression $(\beta=-0.51)$ was statistically significant. These results produce validity evidence for the LSV and are explored in terms of possible clinical implications for the development of strategies to increase happiness.

Keywords: life satisfaction; subjective well-being; depression; anxiety; stress.

\section{RESUMEN - Felicidad e Indicadores de Psicopatología en Brasileños}

Este estudio evalúa las relaciones de satisfacción de vida con depresión, ansiedad y estrés. Tales asociaciones todavía no fueron investigadas de manera conjunta en el contexto brasileño. Los participantes fueron 371 universitarios (64\% mujeres) que respondieron la ESV y la Escala de Depresión, Ansiedad y Estrés. Correlaciones indicaron asociaciones moderadas de satisfacción de vida con depresión $(r=-0,41)$ y asociaciones pequeñas con ansiedad $(r=-0,22)$ y estrés $(r=-0,25)$. Un modelo de regresión múltiple fue utilizado para evaluar el papel predictivo de depresión, ansiedad y estrés sobre la satisfacción de vida. Este modelo predice $17 \%$ de la varianza total, siendo sólo la depresión $(\beta=-0.51)$ estadísticamente significativa. Estos resultados producen evidencias de validez para la ESV y son explorados en términos de posibles implicaciones clínicas para el desarrollo de estrategias de aumento de felicidad.

Palabras clave: satisfacción de vida; bienestar subjetivo; depresión; ansiedade; estrés.

Ser feliz é o desejo mais importante de milhares de pessoas em 47 países (Diener, 2000). Além de muito desejada, a felicidade parece ser algo importante para as pessoas, pois pesquisas evidenciam que ela está associada ao sucesso profissional (Diener, Ng, Harter, \& Arora, 2010), à longevidade, a melhores condições de saúde (Diener \& Chan, 2011) e a menores níveis de psicopatologias (Pelechano, González-Leandro, Garcia, \& Morán, 2013, Zanon, Hutz, Reppold, \& Zenger, 2016). Mais especificamente, felicidade apresenta relação inversa com depressão (Joseph, Linley, Harwood, Lewis, \& McCollam,
2004), ansiedade (Quoidbach, Wood, \& Hansenne, 2009) e estresse (Suh, Diener, \& Fujita, 1996). Considerando essas associações, este estudo busca investigá-las e reproduzi-las, conjuntamente, no contexto brasileiro.

\section{Um Pouco do que Sabemos sobre Felicidade}

Muito se avançou o conhecimento sobre a felicidade desde a Grécia Antiga (McMahon, 2008), sendo que há, literalmente, milhares de publicações científicas sobre o tema anualmente. Periódicos internacionais, com relevante fator de impacto, como Journal 
of Happiness Studies, Journal of Positive Psychology, Social Indicators Research, entre outros, têm devotado seu escopo de interesse às inovações na área. Por exemplo, pesquisas evidenciam que a felicidade apresenta forte componente genético e é bastante associado a fatores de personalidade (Lucas \& Diener, 2010). Esses dados indicam que: (a) pais mais felizes tendem a ter filhos mais felizes e (b) pessoas com baixos escores de neuroticismo e altos escores de extroversão tendem a ser mais felizes. O contrário é verdadeiro em ambos os casos descritos anteriormente, sendo que altos níveis de neuroticismo parecem contribuir, consideravelmente, para o aumento de afetos negativos e à baixa satisfação de vida (DeNeve, \& Cooper, 1998).

Apesar do pouco interesse científico pelo tema até meados da década de 70, a proposta de Ed Diener, que caracteriza a felicidade como um componente cognitivo e outro afetivo, impulsionou muitas investigações sobre o tema. Nessa proposta, o autor e seus colaboradores adotaram, explicitamente, que a felicidade é subjetiva e que os motivos que fazem uma pessoa feliz não são necessariamente os mesmos para outrem. Porém, o ponto, talvez, mais crítico da contribuição de Diener foi a criação de uma escala padronizada com propriedades psicométricas satisfatórias para avaliar o componente cognitivo da felicidade do ponto de vista subjetivo (e.g., Escala de Satisfação de Vida - ESV: Diener, Emmons, Larsen, \& Griffin, 1985). Isso operacionalizou a coleta de dados sobre o tema por pesquisadores de todo o mundo e permitiu a sistematização do conhecimento sobre um fenômeno comum: o bem-estar subjetivo (Diener, Oishi, \& Lucas, 2009). Escalas para avaliar a dimensão afetiva do bem-estar subjetivo foram propostas por Diener e outros (ver Layous \& Zanon, 2014, para uma descrição atualizada de instrumentos e métodos de avaliação da felicidade).

Com o intuito de aumentar o prestígio científico do tema, Diener adotou a nomenclatura "bem-estar subjetivo" para designar a "felicidade", uma vez que o assunto poderia soar pouco digno de investigação para a comunidade científica (Liubomirsky, 2007, comunicação pessoal). As contribuições de Diener et al. foram essenciais para o desenvolvimento da Psicologia Positiva (Seligman \& Csikszentmihalyi, 2000) duas décadas depois nos Estados Unidos da América. O forte apelo da Psicologia Positiva pela necessidade do estudo dos aspectos saudáveis e virtudes humanas ganhou rápida adesão de pesquisadores pelo resto do mundo. No Brasil, a situação não foi diferente, sendo que pesquisadores, como Claudio Hutz, Solange Wechsler, Silvia Koller, Valdiney Gouveya, dentre outros, têm investigado construtos da área desde a década passada.

Com o intuito de garantir a adequada mensuração da felicidade, são necessárias investigações psicométricas variadas, já que resultados dessas avaliações têm sido usados para subsidiar políticas publicas e comparações transculturais (Diener, 2010). Nesse sentido, o acúmulo de evidências de validade para a ESV constitui um esforço necessário para garantir a interpretabilidade dos escores.

\section{Felicidade e Psicopatologia}

A relação entre o fator de personalidade neuroticismo e felicidade indica que pessoas mais instáveis emocionalmente, e que sofrem, relatam ser menos felizes (Zanon et al., 2016). Ademais, sabe-se que pessoas que costumam pensar repetidamente sobre eventos negativos, ou seja, que tendem a ruminar, vivenciam mais afetos negativos (Nolen-Hoeksema \& Morrow, 1991) e apresentam níveis de bem-estar subjetivo inferior a pessoas que não apresentam tendência ruminativa (Zanon \& Hutz, 2012). Uma possível explicação para essa relação é que o sofrimento psíquico decorre da produção de afetos negativos eliciados durante o processo de ruminação (Lyubomirsky \& Tkach, 2004). Na medida em que as pessoas ruminam, elas revivem eventos negativos repletos de angústias, medos e mágoas. A ruminação é o pensamento típico de pessoas depressivas (NolenHoeksema \& Morrow, 1991), sendo que ela também se faz presente em quadros ansiogênicos e de estresse pós-traumático (Lyubomirsky \& Tkach, 2004).

Em relação ao estresse, especificamente, percebe-se achados contraditórios na literatura internacional referentes a sua associação com felicidade. Suh, Diener e Fujita (1996), por exemplo, verificaram relações inversas entre estresse e afetos positivos, sendo que Feist, Bodner, Jacobs, Miles e Tan (1995) não verificaram tal associação. Um estudo mais recente (Schiffrin \& Nelson, 2010) que usou várias medidas de felicidade - inclusive seu aspecto cognitivo - verificou uma correlação moderada entre satisfação de vida e estresse $(r=-0,48)$.

\section{O Presente Estudo}

Uma vez que depressão, ansiedade e estresse constituem transtornos mentais que acarretam considerável sofrimento para muitos, a investigação de indicadores de sinais e sintomatologias desses transtornos (por meio do autorrelato) e suas relações com felicidade pode ser importante para o desenvolvimento de intervenções que visem ao aumento da felicidade. É possível que a redução de alguns sintomas constituam importantes estratégias para o desenvolvimento da felicidade. Na medida em que se obtenha relações inversas de satisfação de vida com depressão, ansiedade e estresse, ter-se-á replicações de achados internacionais. Ademais, considerando que depressão, ansiedade e estresse coexistem com frequência - o que levou ao desenvolvimento do modelo tripartite (Lovibond \& Lovibond, 1995; Lovibond, 1998), este estudo pretende investigar o papel preditivo independente de cada uma dessas variáveis sobre a satisfação de vida.

Hipótese de pesquisa: depressão, ansiedade e estresse apresentarão relações negativas com satisfação de 
vida. Essa hipótese justifica-se pelas relações inversas verificadas na literatura, sendo que o sofrimento psíquico provindo da sintomatologia pode impactar diretamente na percepção sobre a satisfação de vida. Apesar dos resultados contraditórios na literatura sobre estresse e bem-estar subjetivo, espera-se relação negativa porque o indicador de BES utilizado nessa investigação é satisfação de vida apenas, ou seja, seu aspecto cognitivo. Apesar de ser plausível que alguém com sintomatologia de estresse vivencie afetos positivos com alguma frequência (e.g., decorrentes de reconhecimento por lidar bem com pressões e adversidades no trabalho), é improvável uma avaliação geral de que a vida está adequada quando se experiencia situações estressoras (ou sintomatologia de estresse) com alguma frequência. Por isso, acredita-se que sintomas de estresse estarão negativamente associados à avaliação cognitiva do nível de contentamento com vida.

\section{Método}

\section{Participantes e Procedimentos}

Compuseram a amostra deste estudo 370 universitários (64\% mulheres) de uma universidade privada do interior de São Paulo com idades variando entre 17 e 36 anos $(M=22,8 ; D P=6)$. Aproximadamente $78 \%$ da amostra era solteira. Todos os procedimentos seguiram as normas de conduta ética, sendo este projeto aprovado em comitê de ética (CAEE: 39312414.1.0000.5514). Os participantes responderam ao questionário em suas salas de aula. O tempo da aplicação variou entre 15 e 25 minutos.

\section{Instrumentos}

Escala de Satisfação de Vida (ESV: Diener et. al., 1985). Instrumento de autorrelato que avalia o aspecto cognitivo do bem-estar subjetivo. Ela é composta por cinco afirmações em uma escala Likert de 7 pontos (1-discordo totalmente, 2 -discordo, 3 -discordo ligeiramente, 4 -nem discordo, nem concordo, 5 -concordo ligeiramente, 6 concordo e 7 - concordo totalmente) que avaliam os níveis de concordância dos respondentes em relação a amplos aspectos da vida. Alguns exemplos de itens são: "A minha vida está próxima do meu ideal", "Até agora eu tenho conseguido as coisas importantes que eu quero na vida" e "Se eu pudesse viver minha vida de novo, eu não mudaria quase nada". Essa escala está adaptada e apresenta evidências de validade e fidedignidade no contexto brasileiro (Zanon, Bardagi, Layous, \& Hutz, 2014).

Escala de Depressão, Ansiedade e Estresse (DASS-21: Lovibond \& Lovibond, 1998). É composta por um conjunto de três subescalas, do tipo Likert, de 4 pontos. Cada subescala é composta por sete itens, destinados a avaliar os estados emocionais de depressão, ansiedade e estresse. Nessa escala, é pedido ao respondente que indique o quanto cada sentença aplica-se a si mesmo durante a última semana. São dadas quatro possibilidades de resposta de gravidade ou de frequência organizadas numa escala de 0 a 3 pontos. A subescala de depressão avalia sintomas, como inércia, anedonia, disforia, falta de interesse/envolvimento, autodepreciação, desvalorização da vida e desânimo. A de ansiedade avalia excitação do sistema nervoso autônomo, ansiedade situacional, experiências subjetivas de ansiedade. Finalmente, a subescala de estresse avalia dificuldade em relaxar, excitação nervosa, fácil perturbação/agitação, irritabilidade/reação exagerada e impaciência. Essa escala está adaptada e apresenta evidências de validade e fidedignidade no contexto brasileiro (Vignola \& Tucci, 2014).

\section{Análise de Dados e Resultados}

\section{Estatísticas Descritivas e Correlações de Pearson}

Resultados da Tabela 1 indicam que: (a) trata-se de uma amostra com nível médio-alto de satisfação de vida (de acordo com as normas observadas por Zanon \& Hutz, 2014) e baixos níveis de sintomatologia de depressão, ansiedade e estresse (observado a partir das baixas médias e pequenos desvios padrões); (b) satisfação de vida apresenta maior correlação (moderada e inversa) com depressão e menores relações com ansiedade e estresse (pequenas e inversas); e (c) depressão, ansiedade e estresse apresentam correlações moderadas e positivas entre si - o que indica que os conjuntos de sintomas tendem a coexistirem (ou não coexistirem). Nesse caso, especificamente, essas correlações possivelmente devem-se ao baixo nível de sintomas na amostra estudada - o que pode inflar as correlações.

Tabela 1

Estatísticas Descritivas e Correlações de Satisfação de Vida com Depressão, Ansiedade e Estresse

\begin{tabular}{lcccccc}
\hline Variáveis & $(1)$ & $(2)$ & $(3)$ & $(4)$ & $M$ & DP \\
\hline 1. Satisfação de vida & $(0,80)$ & $-0,41$ & $-0,22$ & $-0,25$ & 4,60 & 1,14 \\
2. Depressão & & $(0,89)$ & 0,71 & 0,70 & 0,67 & 0,64 \\
3. Ansiedade & & $(0,84)$ & 0,79 & 0,65 & 0,62 \\
4. Estresse & & & $(0,85)$ & 0,95 & 0,72 \\
\hline
\end{tabular}

Nota. Todas as correlações são estatisticamente significativas $(p<0,01)$. Os valores na diagonal representam os coeficientes alfa das escalas. 


\section{Regressão Múltipla}

Com o intuito de avaliar conjuntamente o papel preditivo de depressão, ansiedade e estresse sobre a satisfação de vida, realizou-se uma análise de regressão linear múltipla. O modelo de regressão indicou que essas variáveis predizem, aproximadamente, $17 \%$ da variância de satisfação de vida, sendo que depressão foi o preditor mais fortemente associado $(\beta=-0.51)$. Esse resultado indica que um aumento de meio desvio padrão na variável depressão está associado a uma redução de um desvio padrão na variável satisfação de vida. Os demais preditores (ansiedade e estresse) não apresentaram significância estatística no modelo e apresentaram coeficientes de regressão pequenos o que indica baixa predição de satisfação de vida.

Tabela 2

Modelo de Regressão para Predição de Satisfação de Vida a Partir de Indicadores de Psicopatologias

\begin{tabular}{|c|c|c|c|c|c|}
\hline \multirow[t]{2}{*}{ Variáveis } & \multicolumn{2}{|c|}{$\begin{array}{c}\text { Coeficientes } \\
\text { Não Padronizados }\end{array}$} & \multirow{2}{*}{$\begin{array}{c}\begin{array}{c}\text { Coeficientes } \\
\text { Padronizados }\end{array} \\
\beta \\
\end{array}$} & \multirow[t]{2}{*}{$t$} & \multirow[t]{2}{*}{$p$} \\
\hline & B & erro padrão & & & \\
\hline Constante & 24,89 & 0,46 & & 53,80 & 0,01 \\
\hline Depressão & $-4,53$ & 0,64 & $-0,51$ & $-7,13$ & 0,01 \\
\hline Ansiedade & 1,41 & 0,75 & 0,16 & 1,87 & 0,06 \\
\hline Estresse & $-0,09$ & 0,65 & $-0,01$ & $-0,13$ & 0,89 \\
\hline \% de variância total & & & & & 0,17 \\
\hline
\end{tabular}

Nota. $\beta=$ indica coeficiente de regressão padronizado

\section{Discussão}

Este estudo investigou as relações entre satisfação de vida com depressão, ansiedade e estresse e avaliou a proporção de variância independente predita por cada um desses indicadores de psicopatologia sobre a satisfação de vida. As correlações negativas observadas suportam uma replicação de achados internacionais, sendo que o modelo de regressão indica depressão como a variável mais associada à (in)satisfação de vida.

Os resultados deste estudo corroboram parcialmente a hipótese de pesquisa proposta que indicava depressão, ansiedade e estresse como importantes preditores de satisfação de vida. A correlação moderada verificada entre depressão e satisfação de vida $(r=-0,41)$ está de acordo com o esperado e indica que pessoas mais tristes e com pouca motivação para viver são mais infelizes (Zanon et al., 2016). Contudo, as correlações pequenas verificadas entre satisfação de vida com ansiedade $(r=-0,22)$ e estresse $(r=-0,25)$ indicam pouca associação entre os construtos - menos de $9 \%$. De qualquer forma, a pequena relação verificada indica uma tendência de que pessoas que vivenciam experiências de ansiedade com alguma frequência e pessoas mais irritáveis e com dificuldade para relaxar tendam a apresentar níveis inferiores de satisfação de vida que pessoas mais calmas, seguras e tranquilas.

Algumas implicações teóricas desses resultados são que a sintomatologia depressiva pode necessitar de maior atenção em intervenções para o aumento da satisfação de vida. Em revisão à teoria da felicidade autêntica, Seligman, Steen, Park e Peterson (2005) indicam que um aspecto essencial da felicidade se deve a existência de razões para viver - o que comumente não é verificado em quadros depressivos. Assim, é possível que o desenvolvimento de propósito de vida, projetos de vida e sentido para viver constituam passos essenciais para que depressivos possam alcançar níveis mais elevados de satisfação de vida. Obter um escore zero de depressão não indica, necessariamente, satisfação com a vida (Crawford \& Henry, 2003) - ela pode ou não estar presente.

Outro aspecto que talvez influencie na maior relação entre felicidade e depressão é o fato de que depressivos tendem a ruminar mais sobre seus estados de humores e eventos negativos (Lyubomirsky \& Tkach, 2004). Uma vez que a ruminação produz afetos negativos (NolenHoeksema \& Morris, 1991) repetidamente ao longo de longos períodos de tempo - enquanto a ruminação ocorre - é possível que estratégias para redução de ruminação possam constituir aliados do aumento da felicidade - via controle de funções executivas e redução de afetos negativos (ver, Cohen, Mor \& Henik, 2015, para estratégias de redução de ruminação).

Outra atividade, comprovadamente, adequada para o aumento da felicidade em pacientes ansiosos e mais estressados é a realização de meditação - o que promove maior ajustamento ao presente (Lyubomirsky \& Layous, 2013). As autoras ainda sugerem que o desenvolvimento de atividades como escrita de cartas de agradecimento, desenvolvimento de otimismo e altruísmo podem aumentar significativamente os níveis de felicidade de forma geral.

Este estudo não está livre de limitações. A primeira delas é a amostra de universitários que não é representativa da população e não permite a generalização destes resultados. Outra limitação deve-se ao uso de autorrelato apenas para avaliação de depressão, ansiedade e estresse. 
O uso de avaliações clínicas e medidas fisiológicas poderia produzir estimativas de psicopatologias mais válidas e fidedignas.

Por fim, espera-se que este artigo alerte e motive estudantes de graduação, pós-graduação e clínicos para a possibilidade do desenvolvimento de aspectos saudáveis no tratamento de seus pacientes. Muito do que se tem buscado na clínica é a redução do sofrimento (o que é extremamente importante), mas talvez muito mais possa ser feito para o aumento do bem-estar dos pacientes. Tais práticas caracterizam a grande contribuição da Psicologia Positiva para as práticas clínicas tradicionais.

\section{Referências}

Cohen, N., Mor, N., \& Henik, A. (2015). Linking executive control and emotional response: A training procedure to reduce rumination. Clinical Psychological Science, 3(1) 15-25. doi: 10.1177/2167702614530114

Crawford, J. R., \& Henry, J. D. (2003). The Depression Anxiety Stress Scales (DASS): Normative data and latent structure in a large nonclinical sample. British Journal of Clinical Psychology, 42(2), 111-31. doi: 10.1348/014466503321903544

DeNeve, K. M., \& Cooper, H. (1998). The happy personality: A meta-analysis of 137 personality traits and subjective well-being. Psychological Bulletin, 124(2), 197-229. doi: 10.1037/0033-2909.124.2.197

Diener, E. (1984). Subjective well-being. Psychological Bulletin, 95(3), 542-575. doi:10.1037/0033-2909.95.3.542

Diener, E. (2000). Subjective well-being: The science of happiness and a proposal for a national index. American Psychologist, 55(1), 34-43. doi: 10.1037/0003-066X.55.1.34

Diener, E. (2012). New findings and future directions for subjective well-being research. American Psychologist, 67(8), 590-597. doi: 10.1037/ a0029541.

Diener, E., \& Chan, M. Y. (2011) Happy people live longer: Subjective well-being contributes to health and longevity. Applied Psychology: Health and Well-Being. 3(1), 1-43. doi: 10.1111/j.1758-0854.2010.01045.x

Diener, E., Emmons, R. A., Larsen, R. J., \& Griffin, S. (1985). The Satisfaction With Life Scale. Journal of Personality Assessment, 49(1), 71-75. doi:10.1207/s15327752jpa4901_13

Diener, E., Ng, W., Harter, J., \& Arora , R. (2010). Wealth and happiness across the world: Material prosperity predicts life evaluation, whereas psychosocial prosperity predicts positive feeling. Journal of Personality and Social Psychology, 99(1), 52-61. doi:10.1037/a0018066

Diener, E., Oishi, S., \& Lucas, R. E. (2009). Subjective well-being: The science of happiness and life satisfaction. Em C. R. Snyder \& S. J. Lopez (Eds.), Oxford handbook of positive psychology (pp. 187-194). Oxford: Oxford University Press.

Feist, G. J., Bodner, T. E., Jacobs, J. F., Miles, M., \& Tan, V. (1995). Integrating top-down and bottom-up structural models of subjective well-being: A longitudinal investigation. Journal of Personality and Social Psychology, 68(1), 138-150. doi: 10.1037/0022-3514.68.1.138

Joseph, S., Linley, P. A., Harwood, J., Lewis, C. A., \& McCollam, P. (2004). Rapid assessment of well-being: The short depression-happiness scale (SDHS). Psychology and Psychotherapy: Theory, Research and Practice, 77(4), 463-478. doi: 10.1348/1476083042555406

Layous, K., \& Zanon, C. (2014). Avaliação da felicidade subjetiva: Para além de dados de autorrelato. Em Claudio Simon Hutz. (Ed.), Avaliação em Psicologia Positiva (pp. 23-42). Porto Alegre: Artmed.

Lovibond, P. F. (1998). Long-term stability of depression, anxiety, and stress syndromes. Journal of Abnormal Psychology, $107(3), 520-526$. doi:10.1037/0021-843X.107.3.520

Lovibond, S. H., \& Lovibond, P. F. (1995). Manual for the Depression Anxiety Stress Scales. Sydney: Psychology Foundation.

Lucas, R. E., \& Diener, E. (2010). Personality and subjective well-being. Em O. P. John, R. W. Robins and L. A. Pervin, (Eds). Handbook of personality (3rd ed., pp. 795-814). New York: The Guilford Press.

Lyubomirsky, S. Comunicação Pessoal, May 17, 2007.

Lyubomirsky, S., \& Layous, K. (2013). How do simple positive activities increase well-being? Current Directions in Psychological Science, 22(1), 57-62. Recuperado de http://sonjalyubomirsky.com/files/2012/09/Lyubomirsky-Layous-20132.pdf

Lyubomirsky, S., \& Tkach, C. (2004). The consequences of dysphoric rumination. Em C. Papageorgiou \& A. Wells (Eds.), Rumination: nature, theory, and treatment of negative thinking in depression (pp. 21-41). Chichester, UK: John Wiley \& Sons.

McMahon, D. M. (2008). The pursuit of happiness in history. Em M. Eid \& R. J. Larsen (Eds.), The science of subjective well-being (pp. 80-96). New York, NY: The Guilford Press.

Nolen-Hoeksema, S, \& Morrow, J. A. (1991). A prospective study of depression and posttraumatic stress symptoms after a natural disaster: The 1989 Loma Prieta earthquake. Journal of Personality and Social Psychology, 61(1), 115-121. doi: 10.1037/0022-3514.61.1.115

Pelechano, V., González-Leandro, P., García, L., \& Morán, C. (2013). Is it possible to be too happy? Happiness, personality, and psychopathology. International Journal of Clinical and Health Psychology, 13(1), 18-24. doi: 10.1016/S1697-2600(13)70003-6

Quoidbach, J., Wood, A. M., \& Hansenne, M. (2009). Back to the future: The effect of daily practice of mental time travel into the future on happiness and anxiety. Journal of Positive Psychology, 4(5), 349-355. doi:10.1016/S1697-2600(13)70003-6

Schiffrin, H. H., \& Nelson, S. K. (2010). Stressed and Happy? Investigating the Relationship Between Happiness and Perceived Stress. Journal of Happiness Studies, 11(1), 33-39. doi: 10.1007/s10902-008-9104-7

Seligman, M. E. P., Steen, T. A., Park, N., \& Peterson, C. (2005). Positive psychology progress: Empirical validation of interventions. The American Psychologist, 60(5), 410-421. doi: 10.1037/0003-066X.60. 5.410

Seligman, M.E.P., \& Csikszentmihalyi, M. (2000). Positive psychology: An introduction. American Psychologist, 55(1), 5-14. doi: 10.1037/0003066X.55.1.5

Suh, E., Diener, E., \& Fujita, F. (1996). Events and subjective well-being: Only recent events matter. Journal of Personality and Social Psychology, 70(5), 1091-1102. doi: 10.1037/0022-3514.70.5.1091

Vignola, R. C., \& Tucci, A. M. (2014). Adaptation and validation of the depression, anxiety and stress scale (DASS) to Brazilian Portuguese. Journal of Affective Disorders, 155(2014), 104-109. doi: 10.1016/j.jad.2013.10.031 
Zanon, C., \& Hutz, C. S. (2012). As relações de afetos positivos e negativos com personalidade, otimismo, esperança, autoestima e satisfação de vida. Em Claudio S. Hutz. (Ed.). Avanços em avaliação psicológica e neuropsicológicade crianças e adolescentes II (pp. 363-388). São Paulo: Casa do Psicólogo.

Zanon, C., \& Hutz, C. S. (2014). Escala de afetos positivos e negativos. Em C. S. Hutz. (Ed.). Avaliação em Psicologia Positiva (pp. 63-68). Porto Alegre: Artmed.

Zanon, C., Bardagi, M. P., Layous, K., \& Hutz, C. S. (2014). Validation of the satisfaction with life scale to Brazilians: Evidences of measurement noninvariance across Brazil and US. Social Indicators Research, 119(1), 443-453. doi: 10.1007/s11205-013-0478-5

Zanon, C., Hutz, C. S., Reppold, C. T., \& Zenger, M. (2016). Are happier people less vulnerable to rumination, anxiety, and post-traumatic stress? Evidence from a large scale disaster. Psicologia: Reflexão e Crítica, 29(1), 1-7. doi: 10.1186/s41155-016-0038-4

\section{Sobre o autor}

Cristian Zanon é mestre e doutor pela Universidade Federal do Rio Grande do Sul e atualmente, é professor auxiliar do Programa de Pós-Graduação em Psicologia da Universidade Federal do Rio Grande do Sul e coordenador do Núcleo de Psicometria. Áreas de interesse em psicometria, avaliação da personalidade e bem-estar subjetivo. 